\title{
CONTRACTILE CELL FORCES EXERTED ON RIGID SUBSTRATES
}

\author{
Joachim Köser ${ }^{1}$, Sebastian Gaiser ${ }^{2}$ and Bert Müller ${ }^{2, *}$ \\ ${ }^{1}$ University of Applied Sciences Northwestern Switzerland, 4132 Muttenz, Switzerland \\ ${ }^{2}$ Biomaterials Science Center, University of Basel, 4031 Basel, Switzerland
}

\begin{abstract}
Adhesive cells including fibroblasts produce contractile forces to the underlying substrate for their locomotion. Such forces are not only high enough to deform compliant membranes such as silicone but also bend rigid microcantilevers. The cell-induced bending of silicon microcantilevers was determined using laser deflection during trypsin treatment. The observed cantilever relaxation corresponds to a contractile cell force of $(16 \pm 7) \mu \mathrm{N}$ per rat-2 fibroblast. The cantilever bending approach represents a unique method for the determination of contractile cell forces on any kind of rigid substrate in desired (physiological) environment. Hence, the fundamental technique allows building cell-based biosensors or should provide a quantitative parameter for characterising the cytocompatibility of load bearing implant surfaces.
\end{abstract}

Keywords: Cell contractility, rat-2 fibroblast, in vitro test, atomic force microscopy, biosensor.

*Address for correspondence:

Bert Müller

Biomaterials Science Center (BMC),

University of Basel

c/o University Hospital Basel

CH-4031 Basel, Switzerland

E-mail: bert.mueller@unibas.ch

\section{Introduction}

Adherent cells deform the underlying substrate as the result of the balance between the cell adhesion and cell contraction, both mechanisms prerequisites for locomotion. Studying cell locomotion, Harris et al. (1980) demonstrated that chicken heart fibroblasts form strong wrinkles on micrometer-thin silicone rubber substrates. Calibrated glass micro-needles served for the determination of the related cell forces of $10 \mathrm{nN}$ per $\mu \mathrm{m}$ (Harris et al., 1980). This fundamental and even quantitative approach, however, has certain restrictions. The wrinkles in the substrate are significantly larger than the cells causing them. The gradually developed wrinkles, which start to interact at rather low cell coverage, exhibit non-linear stress-strain behaviour. Consequently, the technique has inherent limitations with respect to spatial and temporal resolution, although many attempts have been made to master some of them. For example, Lee et al. (1994) have constructed non-wrinkling silicone substrates like a drumhead, which are still free for elastic deformation. The locomoting keratocytes generate deformations, microscopically quantified from the displacements of the latex beads embedded in the silicone membrane. Replacing the silicone by polyacrylamide, the tuning of monomer and cross-linker concentrations allow adjusting the membrane stiffness to the expected contractile cell forces so that one could use the linear range of the overall nonlinear stress-strain behaviour (Pelham and Wang, 1997). Nevertheless, the technique stays semi-quantitative and limited to sub-monolayer cell coverage and specific polymeric materials. Consequently, the method does not account for characteristic in vivo situations, especially because one only detects cell-substrate interactions and the essential cell-cell interactions are neglected.

Besides compliant polymer membranes, rigid silicon pillar structures have been applied for contractile force measurements of individual cells. These measurements include the bending of close-packed pillar arrays, which might be regarded as standing cantilevers, where each individual cell attached bends the pillars at its periphery towards its centre (Petronis et al., 2003; Tan et al., 2003). Such experiments belong to powerful single cell studies, but also do not account for typical in vitro or in vivo conditions. Vertical silicon cantilever bending from parts of individual cells was demonstrated, by means of microtransducers, already more than a decade ago (Galbraith and Sheetz, 1997). Experiments with a collection of cells on rigid substrates commonly used for culturing adherent cells in culture dishes in vitro, or for tissue-integrated load bearing implants in vivo, are basically unknown. Simple estimations confirm that the detection of the contractile forces of a population of adherent cells, by means of 


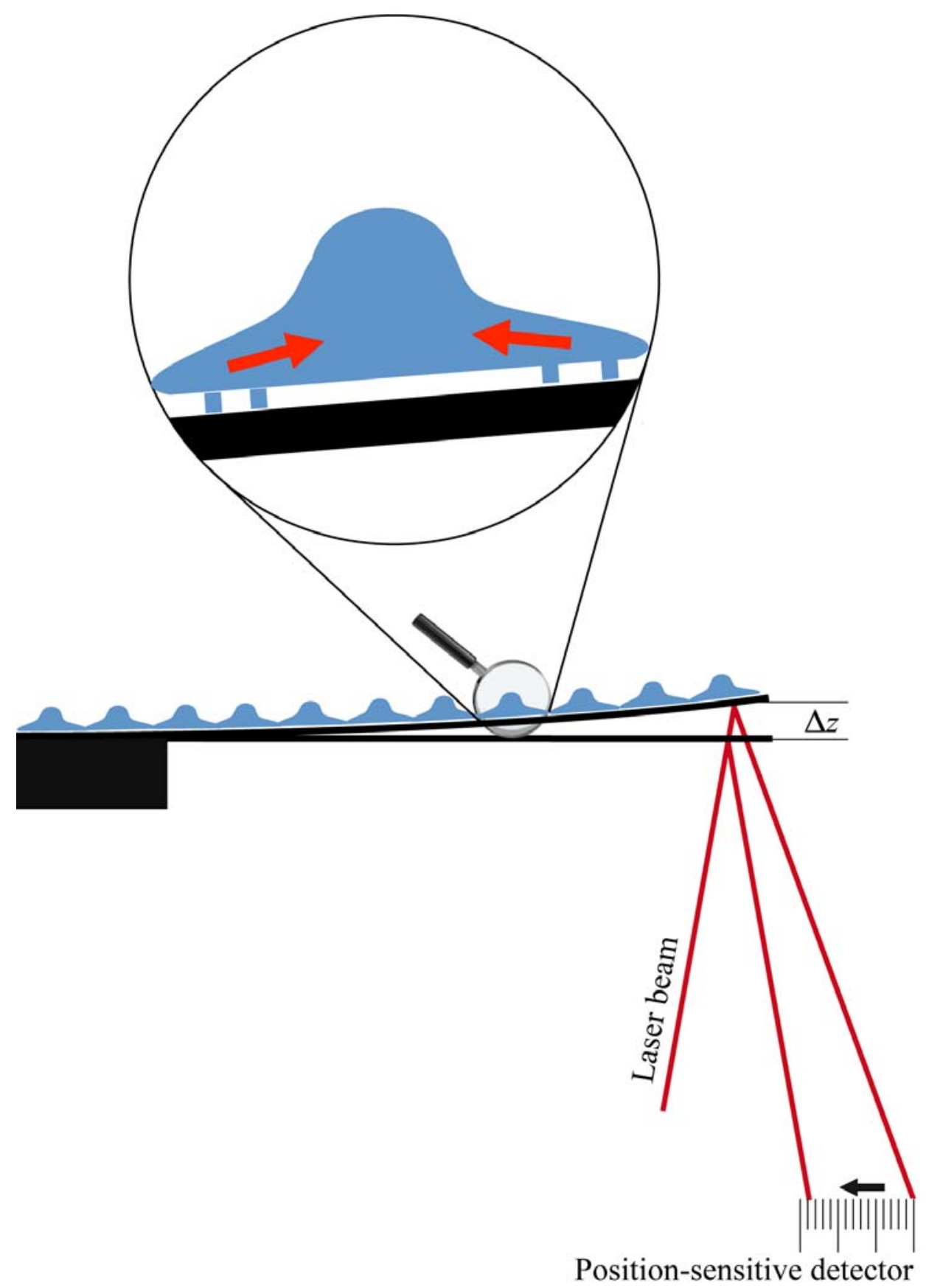

Fig. 1. The scheme illustrates the bending micro-plate method to quantitatively measure the contractile forces of adherent cells on rigid cantilevers detaching the cells using trypsin.

a rigid micro bending plate very similar to a cantilever integrated in an atomic force microscope, should allow the measuring of forces in the pico-Newton-range. Since the contractile forces of fibroblasts are reported to be in the nano-Newton-range, the sensitivity of such a biosensor seems to be well suited for the desired quantification. The authors had to realise, however, that the acquisition of reproducible contractile force measurements depended on the development of an appropriate technique for integral cell force measurements and it took more than a decade before the first, preliminary publication appeared (Köser et al., 2008).

The successful implementation of the bending plate approach could form the basis of a broad variety of fundamental studies on cell-material interactions, biosensor applications and the quantitative characterisation of biomaterials surfaces. All of these would focus on average cell force measurements, which are especially vital for rigid load bearing and dental implants.

\section{Materials and Methods}

\section{Micro-cantilever based cell force measurements}

Fig. 1 illustrates the proposed principle of the contractile cell force measurement. The fibroblasts bind to the substrate by means of their focal adhesions. The cells are known to generate contraction forces acting from their 


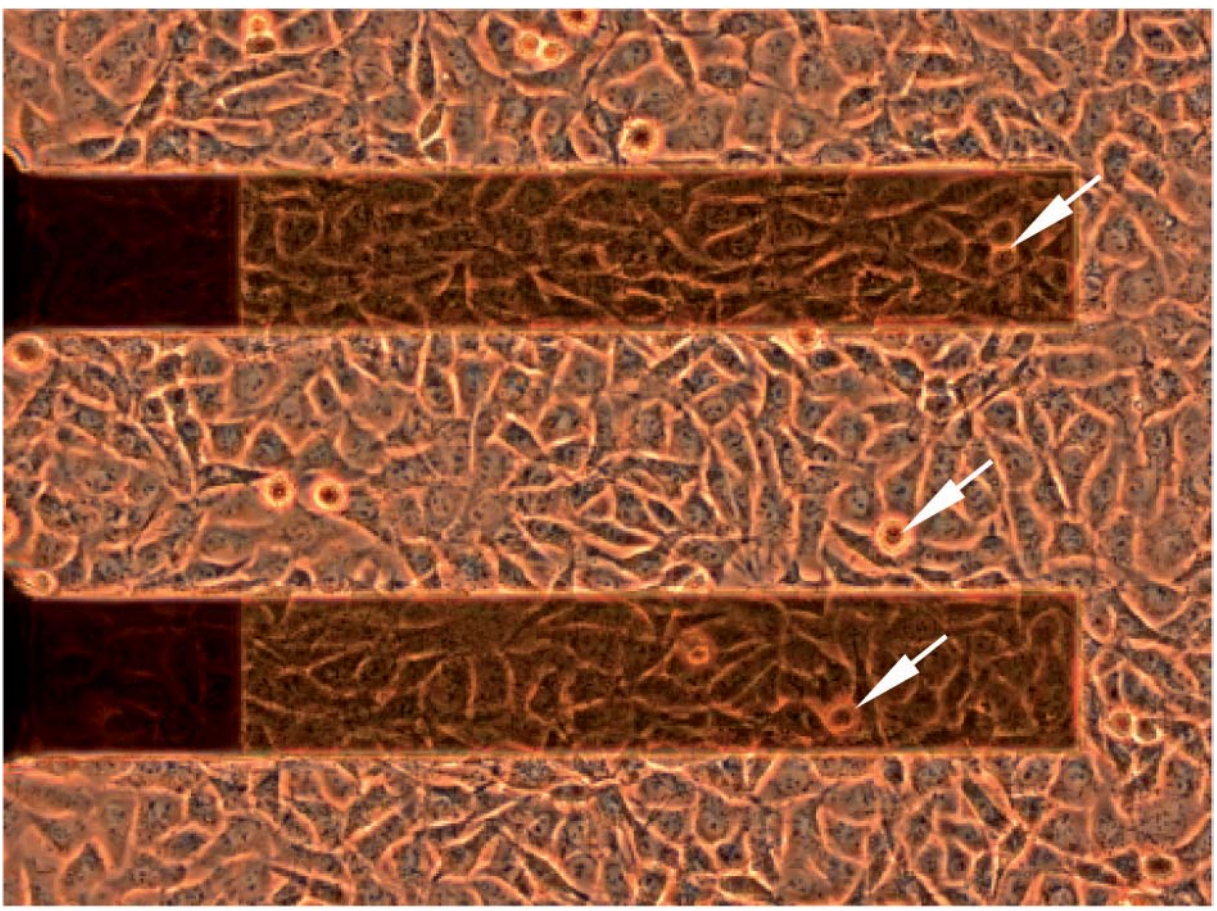

$100 \mu \mathrm{m}$

Fig. 2. The phase contrast micrograph shows rat-2 cells $16 \mathrm{~h}$ after plating on the $1 \mu \mathrm{m}$-thin silicon-cantilevers in a standard culture dish. The fibroblasts on the silicon cantilevers exhibit the same morphological characteristics as their counterparts on the plastic dish. The arrows mark some of the frequently observed mitotic cells present on Si-cantilevers and the polystyrene-dish.

periphery towards its centre, which are accompanied with local substrate deformations. These deformations become detectable, if the substrate is thin enough. Especially for a confluent cell layer, the superposition of the local strain fields gives rise to a bending radius $R$ of the substrate that relates to the integral cell forces. For larger contractile cell forces the bending radius changes to smaller values. For rectangular cantilevers of length $L$, the displacement $\Delta z$ is well approximated by $\Delta z=L^{2} / 2 R$. For example, one can measure the relaxation of the displacement $\Delta z$, while properly detaching the cells from the cantilever as illustrated in the scheme of Fig. 1. Here, the Cantisens ${ }^{\circledR}$ Research cantilever sensor platform (Concentris $\mathrm{GmbH}$, Basel, Switzerland) served for the measurement of the cantilever deflection. This commercial system is based on the laser beam deflection method. The calibration of the on one side gold-coated $\mathrm{Si}(100)$ cantilever was crosschecked by means of the heat test that provided a signal of $100 \mathrm{~nm}$ for a temperature increase of $1 \mathrm{~K}$ (Urwyler et al., 2011).

The task remains to calculate the average contractile force per cell from the observed geometrical alteration. Here, the approaches rely on the Stoney formula (Stoney, 1909) that was derived for the stress generated by ultrathin metallic films on thicker substrates. It correlates the film stress $\sigma_{\mathrm{F}}$ to the mechanical properties of the substrate material, namely the Young's modulus $E$ and the Poisson ratio $v$, and the geometrical parameters bending radius $R$, film thickness $t_{\mathrm{F}}$ and substrate thickness $t_{\mathrm{S}}$ :

$$
\sigma_{F}=\frac{E}{(1-v)} \cdot \frac{t_{S}{ }^{2}}{6 R t_{F}}=\frac{E}{(1-v)} \cdot \frac{t_{S}{ }^{2}}{3 L^{2} t_{F}} \cdot \Delta z \text { (1) }
$$

The applicability of the Stoney formula to the fibroblasts on micrometer-thin silicon substrates is somewhat questionable, because the mean cell height is comparable to or even larger than the cantilever thickness. Note, however, that the stress is directly proportional to the displacement measured, since the other geometrical parameters should be constant as the mechanical properties of the cantilever. This direct relation between integral cell force and displacement allows the adaptation of the cantilever dimensions to reach the necessary precision for the expected contractile cell force. It should be mentioned that even for the wellestablished $\mathrm{Si}(100)$ cantilevers, which were also used in the present study, different values for the Young's modulus and the Poisson ratio can be found in literature. For the present study, the values of W. A. Brantley (Brantley, 1973) were applied: $\mathrm{E}=130 \mathrm{GPa}$ and $v=0.28$.

\section{Cell seeding on micro-cantilevers}

Rat-2 fibroblasts (ATCC CRL-1764) were cultured on standard polystyrene culture dishes (Easy-Grip ${ }^{\mathrm{TM}}$, Becton Dickinson AG, Allschwil, Switzerland) in DMEM (SigmaAldrich, Buchs, Switzerland) supplemented with 10\% FCS (PAA, Chemie Brunschwig, Basel, Switzerland), glutamine (Sigma-Aldrich) and antibiotics (PenicillinStreptomycin, Sigma- Aldrich). The day before the force 
measurements, the fibroblasts were seeded on silicon micro-cantilever arrays with nominal dimensions of $500 \mu \mathrm{m} \times 100 \mu \mathrm{m} \times 1 \mu \mathrm{m}$ (CLA-500-010-08, Concentris) In fact, the cantilevers are about $50 \mu \mathrm{m}$ longer to account for the finite laser beam size. A few of the micro-cantilevers that were used (CLA-750-010-08, Concentris) were $750 \mu \mathrm{m}$ long. All micro-cantilever arrays produced in an array of eight cantilevers contained on one side a coating consisting of the $3 \mathrm{~nm}$-thin Ti adhesion layer and the $20 \mathrm{~nm}$-thick gold layer to achieve the necessary reflectivity for the optical readout of cantilever bending by means of the laser beam deflection method. The fibroblasts adhered on the silicon side of the cantilever, which had been UV/ozone treated for $50 \mathrm{~min}$ (UV clean, model 135500, Boekel Industries Inc., Feasterville, PA, USA) and pre-incubated in cell culture medium for at least $1 \mathrm{~h}$. During the overnight incubation the rat-2 fibroblasts had enough time to appropriately adhere, spread, and generate contractile forces for substrate deformation. Microscopy served to verify the absence of cells on the backside of the cantilevers.

Phase contrast microscopy (IX-71, Olympus, Tokyo, Japan) images as represented in Fig. 2 show a confluent cell layer with equivalent morphology on the polystyrene dish and the micrometer-thin cantilevers indicating a suitable substrate preparation process. After inspecting cell morphology and density, the cell-seeded micro-cantilever arrays were transferred into a liquid flow chamber (flow rate $0.42 \mu \mathrm{L} / \mathrm{s}$ ) of the Cantisens ${ }^{\circledR}$ Research cantilever sensor platform to read-out the cell-induced displacements $\Delta z$ of the up to eight micro-cantilevers, simultaneously. The injection of 1\% trypsin (Sigma-Aldrich) and $3.4 \mathrm{mM}$ EDTA in Dulbecco's Modified Eagle's medium (DMEM) into the flow chamber induced cell detachment and the relaxation of the cantilevers and, therefore, allows deducing the average contractile force per rat-2 fibroblast. Two experienced researchers manually counted the cells on the optical micrographs of the cell-seeded cantilevers to determine the number of cells including error bar.

\section{Cell height measurements}

In order to determine the contractile force per fibroblast from the relaxation measurement, the height $t_{\mathrm{F}}$ has to be estimated. This average cell height, however, is difficult to obtain, since the cells do not form a film of constant thickness such as metal coatings. One can get a rough estimate using confocal laser microscopy, but we decided to apply atomic force microscopy (AFM), which yields much higher accuracy, especially perpendicular to the substrate surface. For this purpose, rat-2 fibroblasts were seeded onto $1 \mathrm{~cm} \times 1 \mathrm{~cm} \mathrm{Si-pieces} \mathrm{cut} \mathrm{from} \mathrm{4-inch} \mathrm{wafers} \mathrm{(4PO/B/5-}$ $10 / 525 \pm 20 / \mathrm{SSP} / \mathrm{TTV}<5$, Siegertcon, Aachen, Germany). After a time period of about $2 \mathrm{~h}$, the cells were formalinfixated. After a residence time of $5 \mathrm{~min}$ the medium was replaced by means of PBS. Subsequently, AFM measurements with a Nanosurf ${ }^{\circledR}$ EasyScan 2 FlexAFM (Nanosurf AG, Liestal, Switzerland) and a sensor element with a spring constant of $0.03 \mathrm{~N} / \mathrm{m}$ were performed. The Easyscope microscope of the Nanosurf system served for the selection of the $100 \mu \mathrm{m}$-wide areas to be scanned in the static force mode. The set point corresponded to $5 \mathrm{nN}$.
256 lines (each consisting of 256 points) were acquired with a speed of $1.3 \mathrm{~s}$ per line. Out of five AFM images the maximal heights $t_{\mathrm{F}}$ of 24 cells were analysed.

\section{Results}

The real-time monitoring of the cell-seeded cantilever in the Cantisens ${ }^{\circledR}$ system reveals that the cell detachment by trypsin injection produces a deflection signal $\Delta z$ that is perfectly described using the function

$$
\Delta z(t)=0.5 z_{0}\left(1-\tanh \left(\frac{t-t_{0}}{\tau}\right)\right)(2)
$$

where $z_{0}$ corresponds to the deflection amplitude, $\tau$ denotes the time constant and $t_{0}$ the injection time by means of the inflection point (see Fig. 3). The detailed experimental setup determines the time constant and the start of the trypsin injection controls the inflection point. At the selected conditions the cells were detached within 50 to $100 \mathrm{~s}$, so that the injection interval of $250 \mathrm{~s}$ was long enough. The only parameter that characterises the total contractile force of the fibroblasts detached from the micro-cantilever is the deflection amplitude. For the data shown in Fig. 3, the deflection amplitude of the $750 \mu \mathrm{m}$ long cantilever corresponds to $1061 \mathrm{~nm}$. The conversion to a $500 \mu \mathrm{m}$-long cantilever, that we generally used, results in a deflection amplitude of $(465 \pm 2) \mathrm{nm}$. The error bar of the deflection amplitude, which is below $1 \%$, was found by means of the Levenberg-Marquardt algorithm from the proFit code 6.1.10 (Quantum Soft, Uetikon am See, Switzerland) that served for fitting the experimental data and for deriving the related errors of the three independent parameters.

The optical micrograph of the cell-seeded cantilever obtained before trypsin injection, as given in the inset of Fig. 3, permits cell counting to determine the cell density and finally the contractile force per cell. The number of cells on this $100 \mu \mathrm{m} \times 750 \mu \mathrm{m}$ cantilever corresponds to $205 \pm 18$, which relates to a cell size of $366 \mu \mathrm{m}^{2}$, a cell density of 2,500 fibroblasts per $\mathrm{mm}^{2}$ and about 39 cells along the $750 \mu \mathrm{m}$-long cantilever. The cells seem to be elongated along the axis of the cantilever, an observation not found for the image in Fig. 2.

Fig. 4 shows the measurements of three cantilevers, where $100 \%, 60 \%$, and $37 \%$ of the cantilever surfaces are covered with a monolayer of fibroblasts and the remaining parts of the micro-cantilever are intentionally empty. The use of a cantilever without cells does not give any detectable relaxation signal. As expected, the cantilever relaxation as the result of the trypsin injection scales with the cell coverage. Only the deflection amplitude is affected, time constant and inflection point stay constant because the setup is identical for the measurements. The optical micrographs show rat-2 fibroblast morphologies well comparable but not identical to the ones observed in Fig. 2. The cells, counted on the cantilever before relaxation, matched $(97 \pm 8),(58 \pm 6)$, and $(35 \pm 4)$ fibroblasts, respectively.

The experiments on confluent cell layers were repeated several times to determine the variations of the contractile 


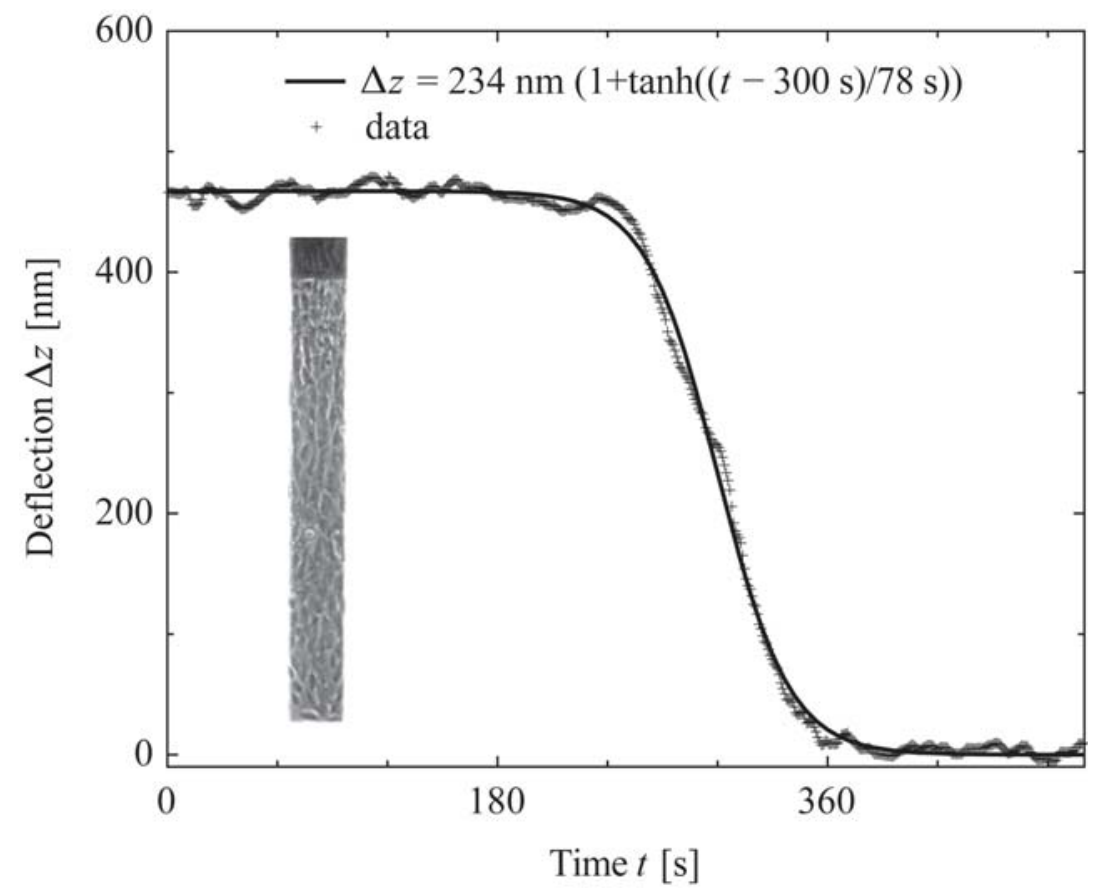

Fig. 3. The cantilever relaxation as the result of trypsin-injection-induced cell detachment can be described by means of a tanh-function that allows fitting the deflection amplitude with high precision. The rat-2 fibroblasts can be imaged before the experiment to derive the bending or displacement per cell (cf. optical micrograph). The injection period of trypsin lasted from $t=240 \mathrm{~s}$ to $t=490 \mathrm{~s}$.

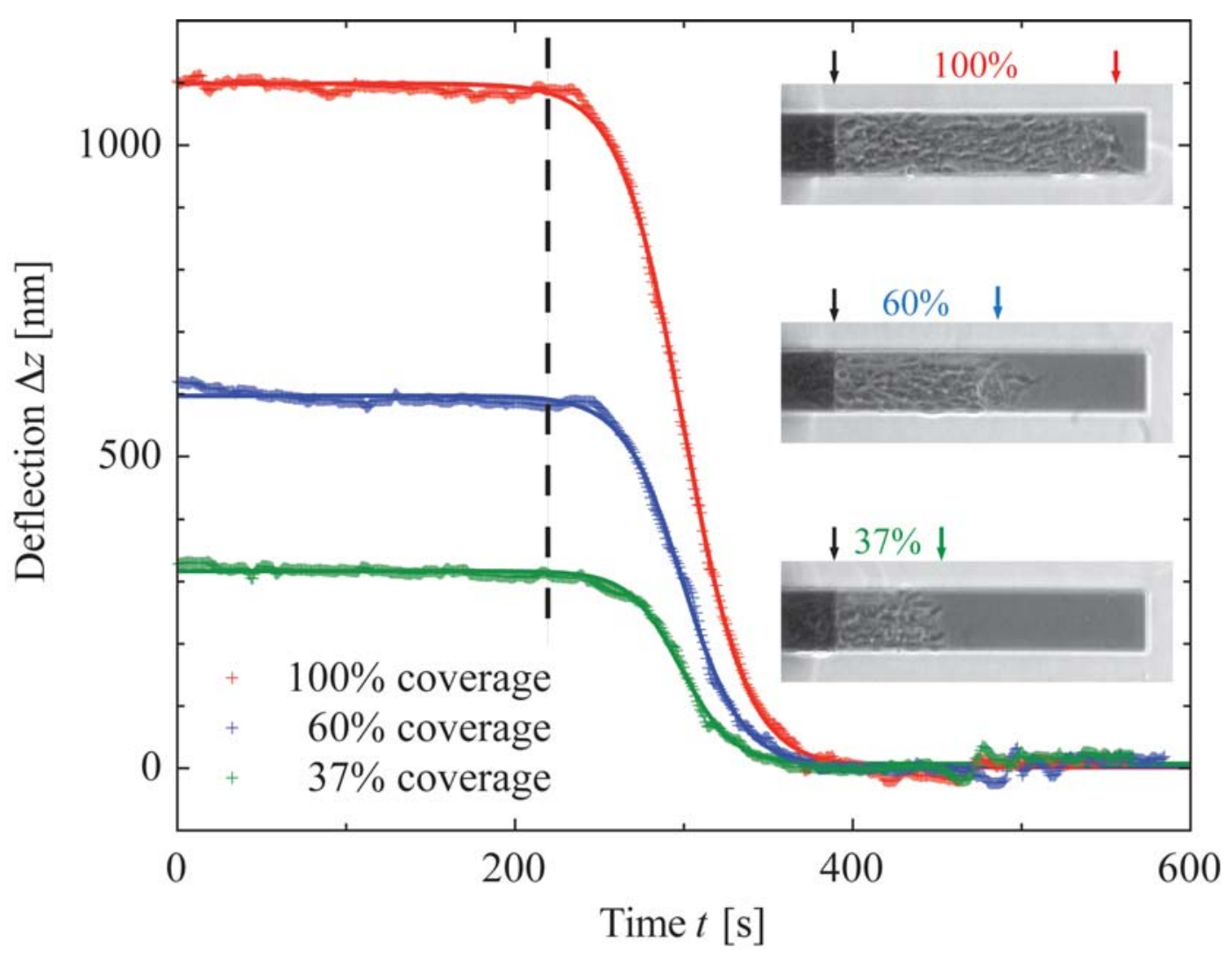

Fig. 4. The cantilever relaxation depends directly on the area covered by the rat-2 fibroblasts. The dashed line points to the start of cell detachment. 
Table 1. To determine the average contractile force per rat-2 fibroblast on Si substrates, the values for cell number on $500 \mu \mathrm{m}$-long cantilevers, the related cell densities, the deflection amplitudes, and the average contractile cell force per experiment are listed.

\begin{tabular}{|c|c|c|c|c|}
\hline Experiment & Cell number & Cell density $/ \mathrm{mm}^{2}$ & $\Delta \mathrm{z} / \mathrm{nm}$ & Cell force / $\mu \mathrm{N}$ \\
\hline$\# 1$ & $141 \pm 2$ & $2560 \pm 40$ & $1094 \pm 2$ & $28 \pm 5$ \\
\hline$\# 2$ & $143 \pm 14$ & $2600 \pm 260$ & $647 \pm 2$ & $16 \pm 5$ \\
\hline$\# 3$ & $141 \pm 11$ & $2560 \pm 200$ & $711 \pm 2$ & $18 \pm 5$ \\
\hline$\# 4$ & $137 \pm 12$ & $2490 \pm 220$ & $465 \pm 2$ & $12 \pm 3$ \\
\hline \#5 & $149 \pm 4$ & $2710 \pm 80$ & $508 \pm 3$ & $12 \pm 3$ \\
\hline$\# 6$ & $134 \pm 6$ & $2440 \pm 110$ & $930 \pm 11$ & $25 \pm 6$ \\
\hline$\# 7$ & $162 \pm 2$ & $2950 \pm 40$ & $1002 \pm 5$ & $22 \pm 4$ \\
\hline$\# 8$ & $168 \pm 20$ & $3050 \pm 370$ & $843 \pm 4$ & $18 \pm 5$ \\
\hline$\# 9$ & $156 \pm 12$ & $2840 \pm 220$ & $944 \pm 7$ & $22 \pm 6$ \\
\hline$\# 10$ & $170 \pm 6$ & $3090 \pm 110$ & $703 \pm 2$ & $15 \pm 3$ \\
\hline$\# 11$ & $142 \pm 7$ & $2580 \pm 130$ & $446 \pm 4$ & $11 \pm 3$ \\
\hline$\# 12$ & $154 \pm 3$ & $2800 \pm 60$ & $794 \pm 3$ & $19 \pm 4$ \\
\hline$\# 13$ & $136 \pm 2$ & $2470 \pm 40$ & $274 \pm 2$ & $7 \pm 2$ \\
\hline$\# 14$ & $181 \pm 2$ & $3290 \pm 40$ & $653 \pm 6$ & $13 \pm 3$ \\
\hline$\# 15$ & $170 \pm 7$ & $3090 \pm 130$ & $460 \pm 4$ & $10 \pm 2$ \\
\hline$\# 16$ & $154 \pm 20$ & $2800 \pm 370$ & $367 \pm 3$ & $9 \pm 3$ \\
\hline$\# 17$ & $141 \pm 7$ & $2560 \pm 130$ & $312 \pm 4$ & $8 \pm 2$ \\
\hline$\# 18$ & $143 \pm 3$ & $2600 \pm 60$ & $403 \pm 3$ & $10 \pm 2$ \\
\hline \#19 & $133 \pm 2$ & $2420 \pm 40$ & $421 \pm 3$ & $11 \pm 2$ \\
\hline
\end{tabular}

fibroblast forces and to derive a meaningful average value. Although the individual measurements exhibited reliable values, giving fittings with a standard deviation below $1 \%$ for 17 out of 19 experiments, the amplitudes of the $500 \mu \mathrm{m}$-long cantilevers varied between 300 and $1100 \mathrm{~nm}$. Table 1 summarises the deflection amplitudes of the cell force relaxation measurements. The average deflection amplitude was found to be $(630 \pm 250) \mathrm{nm}$. Table 1 also includes the cell numbers (average value $150 \pm 14$ ) and related cell densities (average value $2730 \pm 260$ fibroblasts per $\mathrm{mm}^{2}$ ), which relate to a mean cell size of $370 \mu \mathrm{m}^{2}$.

Fig. 5 represents a characteristic AFM-image of the fixated rat-2 fibroblasts on Si-substrates. Because the image shows both the substrate and the individual cells, one can easily derive the maximal height of each cell. For the areas above the cell nucleus the average maximal height was found to $t_{\mathrm{F}}=(3.4 \pm 0.5) \mu \mathrm{m}$.

Using the mechanical properties of $\mathrm{Si}(100)$ $(E=130 \mathrm{GPa}, v=0.28)$ and the geometry of the cantilevers, one obtains the mean contractile force per rat-2 fibroblast from the incorporation of the average deflection amplitude, the average cell density, and the average cell height into eqn. (1). It corresponds to $(16 \pm 7) \mu \mathrm{N}$.

\section{Discussion}

The rat-2 fibroblasts generate a contractile force, which significantly bends the underlying $1 \mu \mathrm{m}$-thin silicon cantilever. The trypsin-induced cell detachment leads to cantilever relaxation. The amplitude of the relaxation signal can be precisely measured usually with an error bar smaller than $1 \%$, as the step-like behaviour is properly described by means of the tanh-function.

The Stoney formula permits the conversion of cantilever bending into the average contractile force per

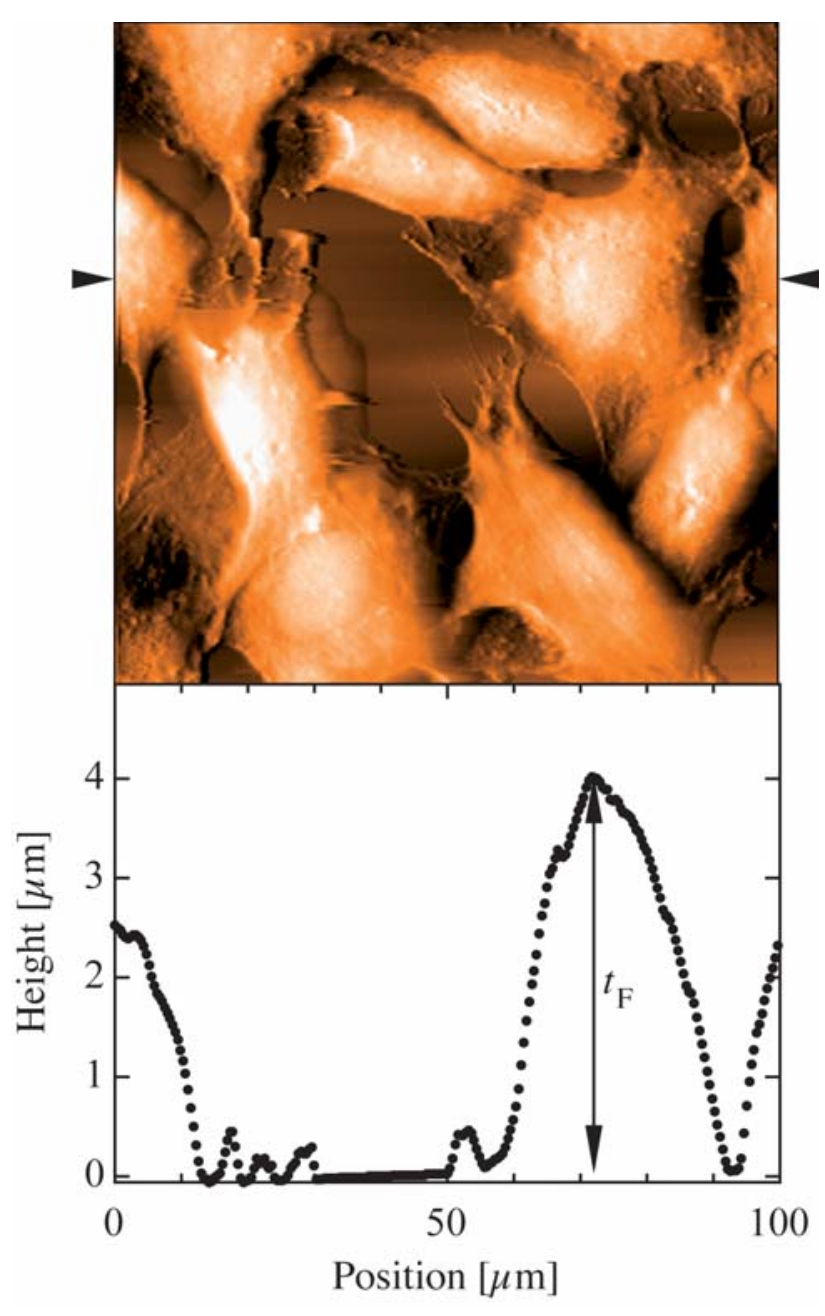

Fig. 5. AFM images of the rat-2 fibroblasts on silicon substrates were used to determine the maximal cell height $t_{\mathrm{F}}$ as indicated by the selected line scan. 
fibroblast provided the cell height is given. The average contractile force per rat-2 fibroblast of $16 \mu \mathrm{N}$ is much higher than values of fibroblasts on a compliant substrate and derived assuming the maximal cell height measured. The cell height measurement performed within the present study is an upper limit yielding the lower limit of the cell force. Therefore, the actual value of the contractile force of rat- 2 fibroblasts on silicon should be even larger than $20 \mu \mathrm{N}$. The derived force is based on the assumption that the cells form a completely homogeneous strain field.

The forces act at the interfaces between cells and substrate. The related stress is known as interface stress and generally given in $\mathrm{N} / \mathrm{m}$. Therefore the reference to thick films might be inappropriate and the interpretation of the experiments by means of interface stress (Sander, 2003; Sander et al., 2008) is better suited. For the interface stress, one finds a value of $0.15 \pm 0.06) \mathrm{N} / \mathrm{m}$. With an average cell extension of $19 \mu \mathrm{m}$, this gives $(2.9 \pm 1.2) \mu \mathrm{N}$ per fibroblast. This approach does not include the cell height. The moments exerted by the fibroblasts onto the cantilever, however, critically depend on the orientation and length of the actin filaments. Further studies on the nanoscopic level are necessary to explain the development of contractile cell forces and to validate the applicability of available approaches.

The contractile cell force on the rigid substrates derived in the present study is several orders of magnitudes higher than those on compliant environments (Freyman et al., 2001a; Freyman et al., 2001b; Zaleskas et al., 2004; Corin and Gibson, 2010). This is most probably not the result of the special character of the rat-2 fibroblasts but rather due to the strongly increased substrate stiffness. For increasing stiffness of compliant substrates, different authors (Lo et al., 2000; Engler et al., 2006) demonstrated that the cell force correlates with the substrate stiffness, which is in keeping with the present result. Traction force studies (Ghibaudo et al., 2008; Saez et al., 2010) on more rigid pillar structures also show this tendency. In the study of Ghibaudo et al. the fibroblasts cover around 150 pillars with maximal force per pillar of $60 \mathrm{nN}$. For the rigid substrates (spring constant above $100 \mathrm{nN} / \mu \mathrm{m}$ ) they found an average force per pillar of $11 \mathrm{nN}$. Consequently, the contractile force of one fibroblast corresponds to 1 to $2 \mu \mathrm{N}$. Preliminary experiments with different cell types on silicon substrates, as recognised in the acknowledgement below, exhibit contractile forces per cell of the same order of magnitude. Such large forces ( 8 to $25 \mu \mathrm{N}$ ) are also exerted by individual pathogenic fungi on aluminium oxide layers (Bechinger et al., 1999).

The noise level is at least two orders of magnitude below the signal detected and cannot explain the variation from experiment to experiment, which corresponds to more than $50 \%$. In light of a recent study (Rajagopalan et al., 2004), however, this result is more than reasonable, as the cell contractility can vary from experiment to experiment by a factor of 25 for individual cells. Because we integrated over about 100 fibroblasts, one could expect variations by a factor of 2.5. Therefore, even larger cantilevers, which carry in the order of 10,000 cells, should be used to reduce the variations to below $25 \%$. Larger cantilevers also have the advantage of reducing edge phenomena that seem to somewhat modify the morphology of the cells located at the periphery of the cantilever with respect to the fibroblasts in the centre of the cantilever. Please note that for the presented cantilever geometry about $30 \%$ of the cells are located at the edge of the cantilever, which possibly influences spreading, migration, and contractility.

The potential applications of the cantilever bending approach measuring contractile cell forces are wide ranging. From a rather fundamental point of view, one can quantify the cell-substrate interactions for any kind of cell type and any kind of rather rigid substrate at low coverage. Increasing the coverage, the cell-cell interactions become more and more important and should be quantifiable considering the cell-substrate interactions at lower coverage. It is hypothesised that changes of the medium around cells and substrate lead to altering of the contractile cell forces in a predictable manner. These changes can include parameters such as temperature and $\mathrm{pH}$-value but also the presence of a certain amount of toxins similar to trypsin used in the present study, so that biosensors can be built on the basis of the method. For this purpose, however, where single usage is required, the silicon cantilever arrays are rather expensive and could be replaced by low-cost polymer cantilever arrays (Urwyler et al., 2011). The most promising applications of the method, however, are intentional substrate modifications including surface morphology and chemistry to optimise load bearing and dental implants.

\section{Conclusion}

Rat-2 fibroblasts on silicon micro-plates exert an average contractile force of 3 to $30 \mu \mathrm{N}$, a value about three orders of magnitudes higher than contractile forces of fibroblasts on compliant substrates. This is a fundamental observation, since cell attachment on rigid culture dishes relates to the daily practice of cell biologists and osseointegration of bony implants is usually desired after surgical treatments. The presented approach for contractile cell force measurements will not only allow for fundamental studies on cell-materials interactions but also realising cell-based biosensors, which are also encouraging for the characterisation of implant surfaces. Therefore, the presented approach will become an important means to optimise the properties of rigid bone implants fabricated from metals, ceramics, and polymers.

\section{Acknowledgements}

The authors thank the Swiss Nanoscience Institute for financial support, Concentris $\mathrm{GmbH}$ for providing the Cantisens ${ }^{\circledR}$ Research system including some consumables as well as J. Althaus and S. Mushkolaj (University of Basel) for supporting the data analysis. U. Pieles and his team (University of Applied Sciences Muttenz) continuously provided technical support, which is gratefully acknowledged.

Preliminary studies of Daniel Bachmann, Lukas Giger, Bekim Osmani, Mario Schmutziger, Thomas Müller, 
Marcel Stillhart, Adrian Lehner, Liliane Hofstetter, Christoph Zillig, Marc Ammann, Roman Randegger and Raoul Enning at ETH Zürich to determine contractile cell forces of $3 \mathrm{~T} 3$ cells, HFF cells, and myotubes were performed in the frame of diploma and semester works between 2001 and 2006 with the support of P. Niederer (Zürich, Switzerland), A. Brunink (St. Gallen, Switzerland), J. Hubbell (Zürich, Switzerland), J. Vörös (Zürich, Switzerland) and their teams. These experimental efforts that resulted in very similar values for contraction forces of cells on silicon substrates to those in the present communication and were not published in any journal are especially acknowledged.

\section{References}

Bechinger C, Giebel K-F, Schnell M, Leiderer P, Deising HB, Bastmeyer M (1999) Optical measurements of invasive forces exerted by appressoria of a plant pathogenic fungus. Science 285: 1896-1899.

Brantley WA (1973) Calculated elastic constants for stress problems associated with semiconductor devices. J Appl Phys 44: 534-535.

Corin KA, Gibson LJ (2010) Cell contraction forces in scaffolds with varying pore size and cell density. Biomaterials 31: 4835-4845.

Engler AJ, Sen S, Sweeney HL, Discher DE (2006) Matrix elasticity directs stem cell lineage specification. Cell 126: 677-689.

Freyman TM, Yannas IV, Pek YS, Yokoo R, Gibson LJ (2001) Micromechanics of fibroblast contraction of a collagen-GAG matrix. Exp Cell Res 269: 140-153.

Freyman TM, Yannas IV, Yokoo R, Gibson LJ (2001) Fibroblast contraction of a collagen-GAG matrix. Biomaterials 22: 2883-2891.

Galbraith CG, Sheetz MP (1997) A micromachined device provides a new bend on fibroblast traction forces. Proc Natl Acad Sci USA 94: 9114-9118.

Ghibaudo M, Saez A, Trichet L, Xayaphoummine A, Browaeys J, Silberzan P, Buguin A, Ladoux B (2008) Traction forces and rigidity sensing regulate cell functions. Soft Matter 4: 1836-1843.

Harris AK, Wild P, Stopak D (1980) Silicone rubber substrata: A new wrinkle in the study of cell locomotion. Science 208: 177-179.

Köser J, Gobrecht J, Pieles U, Müller B (2008) Detection of the forces and modulation of cell-substrate interactions. Eur Cell Mater 16 (Suppl 1): 38 (abstract).

Lee J, Leonard M, Oliver T, Ishihara A, Jacobson K (1994) Traction forces generated by locomoting keratocytes. J Cell Biol 127: 1957-1964.

Lo CM, Wang HB, Dembo M, Wang YL (2000) Cell movement is guided by the rigidity of the substrate. Biophys J 79: 144-152.

Pelham Jr. RJ, Wang Y-L (1997) Cell locomotion and focal adhesions are regulated by substrate flexibility. Proc Natl Acad Sci USA 94: 13661-13665.

Petronis S, Gold J, Kasemo B (2003) Microfabricated force-sensitive eastic substrates for investigation of mechanical cell-substrate interactions. J Micromech Microengin 13: 900-913.

Rajagopalan P, Marganski WA, Brown XQ, Wong JY (2004) Direct comparison of the spread area, contractility, and migration of Balb/C $3 \mathrm{~T} 3$ fibroblasts adhered to fibronectin- and RGD-modified substrata. Biophys J 87: 2818-2827.

Saez A, Anon E, Ghibaudo M, du Roure O, Di Meglio J-M, Hersen P, Silberzan P, Buguin A, Ladoux B (2010) Traction forces exerted by epithelial cell sheets. J Phys: Condensed Matter 22: 194119.

Sander D (2003) Surface stress: Implications and measurements. Curr Opin Solid State Mater Sci 7: 51-57.

Sander D, Tian Z, Kirschner J (2008) Cantilever measurements of surface stress, surface reconstruction, film stress and magnetoelastic stress of monolayers. Sensors 8: 4466-4486.

Stoney GG (1909) The tension of metallic films deposited by electrolysis. Proc Roy Soc A 82: 172-175.

Tan JT, Tien J, Pirone DM, Gray DS, Bhadriraju K, Chen CS (2003) Cells lying on a bed of microneedles: An approach to isolate mechanical force. Proc Natl Acad Sci USA 100: 1484-1489.

Urwyler P, Schift H, Gobrecht J, Häfeli O, Altana M, Battiston F, Müller B (2011) Surface patterned polymer micro-cantilever arrays for sensing. Sensors and Actuators A: Physical, in press (doi:10.1016/j.sna.2010.12.007).

Zaleskas JM, Kinner B, Freyman TM, Yannas IV, Gibson LJ, Spector M (2004) Contractile forces generated by articular chondrocytes in collagen-glycosaminoglycan matrices. Biomaterials 25: 1299-1308.

\section{Discussion with Reviewers}

Reviewer I: Do the authors see a chance to combine the cantilever deflection experiments with an in situ determination of the cell coverage?

Authors: In principle, a microscope can be included into the experimental setup. The Cantisens instrument, however, does not allow the incorporation of a microscope that helps to visualise the cells on the cantilevers. Such a microscope would be extremely supportive, as one can observe the development of the contractile cell forces and the influence of shape changes often related to the functionality of the cells.

Reviewer II: The force measurement was preformed after overnight incubation of the cantilevers with the fibroblasts. For the determination of the maximum cell high the adhesion time was only $2 \mathrm{~h}$. These measurements must be performed after equal incubation times. Please comment. Authors: We have to agree with this comment and regret not applying the same protocol. For our estimation of the cell height the diameter of the cell's nucleus has crucial impact and one can reasonably assume that this diameter will not change between 2 and $24 \mathrm{~h}$. This assumption is supported by several CLSM images, which do not indicate any significant change of cell height within this period of 
time. As a consequence, the estimation of cell height might be somehow imprecise. For the main messages, which are based on the effect of cantilever bending, the precision of this measurement is of secondary importance. We hope that the procedure is described well enough and the reader will tolerate this lack of agreement in the protocols.

Reviewer II: The cell force was determined after overnight adhesion. The forces must be determined at different time points of adhesion. Please comment.

Authors: This suggestion is gratefully acknowledged. We have already tried several times to measure the development of contractile cell forces, but must state - we failed. This might be the result of our inability. Hence, we have to restrict ourselves to the selected time point. For the future, we will select other time points.

Reviewer II: In Fig. 1 it would be helpful to show the angle and radius as used in eqn. (2). Please comment.

Authors: Unfortunately, we could not address this suggestion: eqn. (2) does not contain any radius or angle. The radius $R$ is given in eqn. (1), but we think that it is almost impossible to incorporate it into Fig. 1 in an appropriate way, as it is so large. To introduce another angle seems to be not really helpful. The deflection signal $\Delta z$ is the direct output of the Cantisens system and can be easily converted into $R$ using the formula in the text, since the cantilever length is known. 